\title{
On Whether the Higher-Order Thought Theory of Consciousness Entails Cognitive Phenomenology, Or: What Is It Like to Think That One Thinks That P?
}

\author{
Richard Brown \\ CUNY LaGuardia \\ Pete Mandik \\ William Paterson University
}

\begin{abstract}
The question at the center of the recent growing literature on cognitive phenomenology is this: In consciously thinking $\mathrm{P}$, is there thereby any phenomenology? In this paper we will present two arguments that "yes" answers to this question follow from the Higher-Order Thought (HOT) theory of consciousness, especially the version articulated and defended by David Rosenthal. The first, the general argument, aims to show that on the HOT theory all phenomenology is cognitive. The second, the central argument, aims to show that all conscious thoughts have phenomenology.
\end{abstract}

Among our conscious states are conscious thoughts. The question at the center of the recent growing literature on cognitive phenomenology (e.g., Bayne and Montague 2011; Pitt 2004) is this: In consciously thinking P, is there thereby any phenomenology? One way of clarifying the question is to say that it concerns 
whether there is any proprietary phenomenology associated with conscious thought. Is there any phenomenology due solely to thinking, as opposed to phenomenology that is due to some co-occurring sensation or mental image? The question here concerns whether there is any phenomenology that attaches to the thinking process itself as opposed to cases of sensation and perception. Thinking in this sense will include such things as wondering, understanding, entertaining, doubting, etc. We will focus on occurrent thoughts in what follows but we take it that the argument generalizes to all instances of cognition.

An additional way of clarifying the question is in terms of the "what it's like" and "something it's like" phraseology central to much work on consciousness since Nagel (1974). ${ }^{1}$ In having a conscious thought, or in consciously thinking, is there thereby something it is like for one to be in that state or to do that thinking? 2 Further, is what it is like for one to have that thought not due to some co-occurring noncognitive state such as a sensation or an image?

Another question linked in the literature to this central question is whether conscious thoughts differing in their content differ in their respective associated phenomenology. If $\mathrm{P}$ and $\mathrm{Q}$ are distinct propositions, is what it's like to consciously think P different from consciously thinking Q?

In this paper we will present two arguments that "yes" answers to these questions of cognitive phenomenology follow from the Higher-Order Thought (HOT) theory of consciousness, especially the version articulated and defended by Rosenthal $(2005 ; 2011)$. Call these two arguments (until we come up with better names) the general argument and the central argument.

\section{THE GENERAL ARGUMENT: ALL PHENOMENOLOGY IS COGNITIVE}

The goal of Rosenthal's HOT theory is to explain consciousness. Of course, the word "consciousness" encompasses a wide variety of phenomena. Fortunately, more can be said to narrow down the explanatory targets of the theory. First and foremost in Rosenthal's exposition, HOT theory offers an explanation of what he calls "state consciousness." State consciousness is a mental state's being conscious. Many theorists and nontheorists alike accept that some mental states are conscious and that other mental states are unconscious. In accepting this, they thereby accept a claim that can be made in terms of state consciousness, and there is ample empirical and commonsense data to suggest that we ought to accept this distinction.

A secondary explanatory target of HOT theory is there being something it's like for one to be in such-and-such a mental state. For want of a better name, call this explanatory target "what-it-is-likeness." Some theorists may view these explanatory targets, state consciousness and what-it-is-likeness, as one and the same. This may very well be something that, for instance, Nagel has in mind when 
he writes that "an organism has conscious mental states if and only if there is something that it is like to be that organism" (Nagel 1974, 436). Whether Rosenthal would go along with such an identification is not entirely clear. Nonetheless, at a minimum the explanatory targets are verbally distinguishable, and Rosenthal's expositions of HOT theory aim to explicitly address both.

The core of the HOT-theoretic explanation of state consciousness is that a mental state's being conscious just is one's having a (suitable) thought that one is in such-and-such mental state. This thought, being a mental state about oneself as being in some other mental state, is thus higher order. There are further wrinkles to Rosenthal's explanation of state consciousness, but they are inconsequential to the present paper. Having the core stated as above will suffice for the points to be made.

One line of reasoning that Rosenthal gives for this explanation of state consciousness hinges on the claims that (1) one's conscious states are mental states of which one is conscious and (2) the relevant way in which one is conscious of one's own mental states is by thinking about them (as opposed to, for instance, perceiving, imagining, or having sensations of them). Again, there are details of Rosenthal's exposition that we are omitting so as to call attention to the core and its relation to key ideas in the cognitive phenomenology literature. Notice, in particular, the key role that is played by cognitive states — thoughts, especially higher-order ones—in explaining state consciousness.

Another line of reasoning that Rosenthal gives for his explanation of state consciousness hinges on claims concerning the way one's own mental states appear to one. Key here are claims that (1) a state's being conscious is its appearing to one that one is in such-and-such mental state, and (2) the relevant way in which one is appeared to is via thought-it appears to one that one is in such-and-such mental state when one (in some suitable way) thinks (as opposed to senses or imagines) that one is in such-and-such mental state. Again, notice the key role that a cognitive state is made to play in Rosenthal's account. The relevant kind of appearing is a cognitive kind of appearing - its appearing that $\mathrm{P}$ just is one's thinking that $\mathrm{P}$.

It is natural to conclude from the foregoing that thinking thoughts with different contents gives rise to different appearances. Where $P \neq Q$, (1) in thinking that $\mathrm{P}$ it appears that $\mathrm{P},(2)$ in thinking that $\mathrm{Q}$ it appears that $\mathrm{Q},(3)$ the thought that $\mathrm{P}$ is not the thought that $\mathrm{Q}$, and (4) its appearing that $\mathrm{P}$ is not its appearing that $\mathrm{Q}$.

This cognitive kind of appearing does double duty for Rosenthal. Not only does it play a role in Rosenthal's explanation of state consciousness, it also plays a role in Rosenthal's explanation of what-it-is-likeness. This latter point is evident in the following passage from Rosenthal.

Rosenthal (2011) writes:

As many, myself included, use that phrase, there being something it's like for one to be in a state is simply its seeming subjectively that one is in that state. Indeed, Block $(2011,424)$ quotes me to that effect: "What it is like for one to have a pain, in the relevant sense of that idiom, is 
simply what it is like for one to be conscious of having that pain" (Rosenthal 1997, 733). And on that construal of 'what it's like', the theory does hold that a HOT is sufficient for there to be something it's like for one to be in the state the HOT describes, even if that state doesn't occur. (433-34)

There are three items to note about the quoted passage. The first is that the HOT alone suffices for what-it-is-likeness. This is made especially clear by mentioning cases in which the HOT is about a mental state that doesn't actually exist. Even when the HOT is in this sense "empty," the HOT suffices for there to be something it is like.

The second is that Rosenthal is appealing to a very specific notion of the 'what it is like for one' phrase that he means to contrast with the way it is used by those like Block. The main difference is that on Block's usage, at least as Rosenthal sees him using it, what-it-is-likeness is automatically a property that first-order states have and thus understanding it that way already biases the discussion against the higher-order thought theory, which has as a central tenet that there are first-order states that occur unconsciously and thus for which there is nothing that it is like for the creatures that instantiate those states. For the purposes of the argument of this paper we intend all talk of 'phenomenology' and 'what it is like for one' to be interpreted in the way that the higher-order theory requires.

Finally — and this is a third item to note about the quoted passage - this sufficiency claim about HOTs is a key commonality between HOT theory and one of the central theses defended by cognitive-phenomenology proponents: A thought can give rise to there being something it's like, and does not do so in virtue of there being some co-occurring noncognitive state. If what-it's-likeness and phenomenology are one and the same, then it follows from HOT that all phenomenology is cognitive, in the sense that whenever there is something it's like, this is fully explained by the presence of a certain cognitive state, a higher-order thought. When there is a co-occurring noncognitive state, as when a higher-order thought that one has a sensation of pain is true and there is such a sensation, the sensation is, strictly speaking, entirely irrelevant to the phenomenology that arises.

A clarification is in order: The sense in which all phenomenology turns out to be cognitive needs to be distinguished from the sense of "cognitive phenomenology" that is central to the recent cognitive phenomenology literature. In the literature, the central question is whether there is phenomenology when one consciously thinks that P. Call this the central sense of "cognitive phenomenology." On the HOT theory under consideration, in consciously experiencing pain one need not have cognitive phenomenology in the central sense, since one need not be having a conscious thought. The HOT that makes it the case that one is consciously experiencing pain need not itself be a conscious thought. One has a conscious second-order thought only when one is in a third-order state, and such a third-order state is inessential for simply consciously experiencing pain. Nonetheless, when one has any phenomenology, the phenomenology is due entirely to the presence of a cognitive state and not to any co-occurring noncognitive states. 
The conclusion that we've argued for so far is that (assuming the HOT theory) all phenomenology is cognitive in the sense that phenomenology is explicable fully in terms of cognitive states. What we have not yet addressed, but turn to now, is the question of whether conscious thoughts themselves have any phenomenology. This is to address the central sense of "cognitive phenomenology" in the cognitive phenomenology literature. In the next section, we will present an argument for the conclusion that all conscious thoughts have phenomenology. Before turning to the argument for that conclusion concerning all conscious thoughts, we want here to briefly make a point about a limited class of conscious thoughts, specifically, certain conscious second-order thoughts.

Consider the following scenario in which a person has three mental states: The person has (1) a first-order state, a pain sensation; (2) a second-order state, a thought about the pain sensation; and (3) a third-order state, a thought about the second-order state. This is the sort of scenario that Rosenthal $(2005,110)$ takes to be exemplified in instances of introspective consciousness. In a case of a conscious state that is not introspectively conscious, the state is accompanied by a HOT, but the HOT itself is not conscious. In contrast, on Rosenthal's account of introspective consciousness, in a case of a conscious state that is introspectively conscious, the state is accompanied by a conscious HOT, and the HOT's being conscious requires that it be accompanied by a third-order state. Rosenthal's appeal to the different roles played by conscious and nonconscious HOT's is designed to account for the following apparent difference between introspected and unintrospected conscious states: "When we introspect a state, we are conscious of it in a way that seems attentive, focused, deliberate, and reflective. When a state is conscious but not introspectively conscious, by contrast we are conscious of it in a way that is relatively fleeting, diffuse, casual, and inattentive" (Rosenthal 2005, 110).

When, as in the scenario under consideration, one is introspectively conscious of a pain, it seems natural to suppose that there is indeed phenomenology. Further, ex hypothesi, there is in this scenario a conscious thought, namely, the second-order state. As is the case generally on the HOT theory, all of the phenomenology present is due solely the contributions of cognitive states. And further, what we have in the present scenario is a case in which the conscious state (the second-order state) itself is a cognitive state. So, at least some thoughts have phenomenology, namely, the conscious HOTs that go along with introspectively conscious sensory states. What remains to be addressed is whether nonintrospectively conscious thoughts have phenomenology. We turn to this now in the next section.

\section{THE CENTRAL ARGUMENT: THE PHENOMENOLOGY OF COGNITION}

Suppose that the HOT theorist affirms that (1) in consciously experiencing pain there is phenomenology but denies that (2) in (nonintrospectively) consciously 
thinking $\mathrm{P}$ there is phenomenology. This would seem to give rise to a puzzle, one that we see no happy way for the HOT theorist to resolve.

To bring out the puzzle we can imagine a creature who has plenty of mental states but who has never had a conscious mental state. This creature will have states that have qualitative character (unconscious pains, unconscious seeings of red, etc.) as well as states that have mental attitude and intentional content (unconscious thoughts). The creature feels pain, senses red in its environment, and thinks that it is raining but it never does any of this consciously. This means, ex hypothesi, that it has never had the right kind of higher-order thoughts and so there is nothing that it is like for this creature to have these sensations or thoughts (there being something that it is like for one consists in having the right kind of higher-order awareness of oneself as being in those states). It has no mental appearances. Now suppose that this creature comes to have the right kind of higher-order state about its firstorder pain sensations. It will suddenly go from being a zombie-there being nothing that it is like for it-to experiencing conscious pain in all of it painfulness. Now, though, suppose that it comes to have the right kind of higher-order state about its thought that it is raining. If we deny cognitive phenomenology then we have to say that, though the thought is conscious, there is nothing that it is like for this creature to consciously think the thought. For all intents and purposes it is still a zombie. But the two higher-order states are in every relevant respect the same. They each characterize the creature's mental life as including first-order (world-directed) mental states, and they each describe those mental states in terms of the kinds of properties they exemplify by employing concepts in the content of the higher-order state. In each of the two cases the creature's mental life appears to itself as being a certain way. In the one case it appears as though it is in pain, and in the other it appears as though it is thinking that it is raining. What could explain the existence of phenomenology in the one case and its nonexistence in the other case?

One might be tempted to think that there is simply a difference in the kind of property that one attributes to oneself and that is all that one needs to explain the difference. In the case of a conscious pain, I attribute to myself a state with a certain qualitative character, and since I am conscious of that state in respect of this painful qualitative character what it is like for me is painful. In the case of thoughts, I am not conscious of them as having qualitative character, and so there is nothing that it is like for me to be conscious of them. Rather I characterize them in terms of their mental attitude and intentional content and since these are not qualitative properties there will be nothing that it is like for me to ascribe them to myself.

But this solution won't work. It is true that we are not aware of first-order thoughts as having qualitative character on par with pains and sensations of blue, but we are aware of them in respect of their intentional properties and in respect of their mental attitude. So if I have a higher-order thought to the effect that I am thinking that $\mathrm{P}$, it should appear to me as though I think that $\mathrm{P}$. This is because the content of the higher-order state determines the way my mental life appears to me and if consciousness is mental appearances then what it is like for me should be like 
thinking that P. So we can grant that what it is like to consciously think that $\mathrm{P}$ will be different from what it is like to have a conscious pain. What it is like to consciously have these mental states will differ precisely because I attribute to myself different states with different properties. At most, then, that we attribute distinct kinds of properties to ourselves gives us a reason to think that what it is like for one to have these conscious mental states will differ from each other, but it gives us no reason to think that there will be no phenomenology in one case.

This cognitive phenomenology can be seen as proprietary in the sense that it is distinct from the qualitative character of any sensory mental states. We characterize first-order thoughts in terms of mental attitude (wondering, desire, thought, etc.) and their intentional content ('that p', 'that q or r', etc.), as opposed to their sensory qualitative character. So when I consciously think that $\mathrm{P}$, it will appear to me as though I am thinking that P, when I consciously wonder whether P will appear to me as though I am wondering whether P, etc. If what-it-is-likeness is truly explainable in terms of mental appearances then these mental appearances must result in phenomenology. We now have an explanation for why the phenomenology of cognition is distinct from any sensory phenomenology. This is because the kind of property that appears in one's mental life is an intentional property ascribing an intentional content and a mental attitude held toward that content, rather than a qualitative one, to my mental life.

It is also easy to see why the view is committed to distinct phenomenology for distinct conscious thoughts (where $\mathrm{P} \neq \mathrm{Q}$, the conscious thought that $\mathrm{P}$ and the conscious thought that Q). This is because what it is like for one, on the higherorder thought theory of consciousness, is determined by the exact contents of the higher-order state. So if one represents oneself as thinking that P as opposed to Q we should expect that one's conscious thought will be like thinking that $\mathrm{P}$ whereas the other will be like thinking that Q for the subject of these thoughts.

Though perhaps more tendentious, it is also possible to see another connection to the cognitive phenomenology literature. David Pitt argues that cognitive phenomenology is individuative in the sense that "a thought's having a particular representational content is its having a particular phenomenology" (Pitt 2004, 5). Consider the 'particular representational content' of a typical HOT. Suppose that it is a HOT to the effect that I am in a red* state. Then phenomenologically what it is like for me is like seeing red, and this just is my having a certain representational content in my HOT. So, too, when I consciously think that P I have a HOT to the effect that I am thinking that $\mathrm{P}$ and what it is like for me is like thinking that $\mathrm{P}$. Further, this is completely determined by the representational content of the HOT. Thus we might translate Pitt's claim into higher-order language as "an appropriate higher-order thought's having a particular representational [i.e., intentional] content is its having a particular phenomenology."

That concludes our presentation of the central argument. We now turn to evaluating some common objections and to elaborating on the consequences of denying the conclusion of the central argument. 
Some may object that the very notion of cognitive phenomenology is misguided or confused. We can make sense of there being something that it is like for one to have a conscious pain, but, so the objection continues, what sense can we make of there being something that it is like for one to have a conscious thought? According to this line of thought it is illegitimate to apply the notion of phenomenology to conscious thoughts. We here take no stand on this issue, though one of us is sympathetic to the cognitive phenomenology movement (Brown 2007). Whatever one's ultimate feelings about cognitive phenomenology are, that issue is orthogonal to the one we are here trying to focus on. The issue is not whether one antecedently accepts that there is cognitive phenomenology or not. Rather, the issue is how the higher-order thought theory of consciousness can explain why the mental appearances involving first-order cognitive states do not result in phenomenology whereas the mental appearances involving first-order sensory states do. Thus if one is inclined to see the very idea of cognitive phenomenology as misguided or confused, then one would take our argument as showing that the higher-order thought theory of consciousness is deeply misguided or confused because it is committed to cognitive phenomenology.

A related objection is to insist that we restrict the terms 'phenomenology' and 'what it is like for one' to cases where the higher-order thought employs concepts describing states with qualitative character. But without giving a justification for such a restriction, the debate over whether cognition has phenomenology threatens to devolve into a merely verbal dispute or, worse, to reveal those who deny it as eliminativists about consciousness. Recall the quote from Rosenthal from earlier. He says, quoting his earlier self,

What it is like for one to have a pain, in the relevant sense of that idiom, is simply what it is like for one to be conscious of having that pain. (Ibid.)

Now suppose we replace 'pain' with 'thought' then we have,

What it is like for one to have a [thought], in the relevant sense of that idiom, is simply what it is like for one to be conscious of having that [thought].

There is no reason that stems from the higher-order theory itself that indicates that we should accept the first quote about pains but reject the second quote about thoughts. What principled reason is there to treat these cases differently? If that really is the relevant sense of the idiom then Rosenthal cannot deny that there is something that it is like for one to have a conscious thought. So his own theory does not allow him to restrict the 'what it is like' terminology in this way.

He may insist that what it is like for one involves no phenomenology since that is a property that only qualitative states can have. That is, he may deny the connection between what-it-is-likeness and phenomenology. Many will find this move implausible. The reason for this is that the standard usage of these terms equates the two, and this is even allowing that there is a wide divergence in interpretations 
of the phrase. ${ }^{4}$ And there is good reason for this usage. When we use terms like 'what it is like for one' we mean to isolate those properties of your experience that are felt. We can all agree that there is a lot of information processing going on in my nervous system, but it is also obvious that this information processing is not 'done in the dark' (though some of it is). So if there is something that it is like for one to be in a state then we can conclude that the state has phenomenal properties, those properties in virtue of which there is something that it is like for one to be in the state. If one rejects this line of reasoning then it seems that one is stubbornly choosing to use words in a difficult way, in which case we can find a new term for what thoughts and pains have in common. If one denies that the issue is merely a verbal one about what we call the property that conscious thoughts exemplify then one is revealed as an eliminativist consciousness for the following reasons.

On the view in question, where we distinguish what-it-is-likeness from phenomenology and agree that conscious thoughts have the former but not the latter, the only difference between the two higher-order thoughts (that is, the thought about the first-order thinking and the thought about the first-order sensing of red) is in the intentional content of those thoughts. We then end up with a dilemma. If one is a realist about phenomenology then one will end up with the position that there is something special about the concepts that we employ in higher-order thoughts about sensations that is missing from the thoughts we employ about thoughts. Whatever the difference is, it results in real phenomenology in one case but not in the other. This is in effect to give up the higher-order theory as an explanation of what phenomenology is. It is not merely mental appearances; it is a special kind of mental appearances. The central virtue of higher-order theories lies in their claim to be able to give an explanation of what consciousness is and so to give that up would be a severe blow to the theory.

However, if it is merely a choice about how to use words, something to the effect that ordinary usage restricts terms like 'phenomenology' to sensations, then we cannot be realists about the phenomenal consciousness of pains. ${ }^{5}$ As we have seen, the objector has already admitted that there is no real difference between the two kinds of higher-order states except for the intentional content that the two states have. They have also admitted that in both cases there is something that it is like for the creature to think the thought or have the pain. If all there is to the phenomenology of conscious pain is that it is one of those properties that gets called by a special name in honor of traditional usage then the 'phenomenology' of pain is not what it is cracked up to be. Surely whether one says that a pain is consciously felt as painful or that there is painful phenomenology is not merely a matter of word choice! Conscious pains and sensations of red have something in common with each other that we try to pick out by saying that in each case there is phenomenology. The issue under consideration here is whether conscious thoughts share that property or not. One would not suppose that one could settle the issue of whether whales were mammals or not by appealing to common usage of 'mammal', so why should we expect that to work in this case? 
Further, the proposal to restrict the use of "phenomenology" only to cases in which HOTs deploy concepts of sensations runs into a puzzle. Consider a comparison between two creatures that differ in that one has a third-order HOT and the other doesn't. The first creature, suppose, has a red sensation, a second-order HOT about that red sensation, and a third-order HOT about the second-order HOT. The second creature, in contrast, has only a red sensation and a second-order HOT about it-it lacks a third-order HOT about its second-order HOT. Suppose that the content of the third-order HOT is something along the lines of "I am thinking that I'm having a red sensation." As such, the third-order HOT deploys concepts both of sensations and of cognitions. Question: In virtue of having the third-order state, is there any phenomenology? If the answer is "yes, because the presence of a concept of a sensation in a consciousness conferring HOT suffices for phenomenology" then the phenomenology in question is cognitive phenomenology in the central sense since the state that's conscious in virtue of the third-order state is a thought. Thus the proposal under consideration wouldn't work to avoid using the term 'phenomenology' to describe thoughts. If, instead, the answer to the question is "no, the presence of concepts of sensations in consciousness-conferring HOTs is a necessary but not sufficient condition for phenomenology," then the HOT theorist owes some explanation of why the condition just described wouldn't be sufficient. And as yet, no such explanation has been given.

The central concern here is that if one denies that there is cognitive phenomenology but maintains that there is sensory phenomenology then we need to know what explains this difference. When one looks at the HOT-theoretic explanation one is hard-pressed to point to anything in the machinery of the theory that could account for this difference. As argued above, both HOTs about thoughts and HOTs about sensory states employ intentional contents that determine the way one's mental life appears to one. If it is only in the application of concepts about sensory states that we get real phenomenology, then it must be the case that either there is something special about first-order qualitative states or something special about the kinds of concepts that we use to pick those first-order states out. Either of these options spells doom for the higher-order explanation of consciousness.

Consciousness, on this view, is simply it appearing to one that one is in some first-order state or other. Rosenthal has insisted on this time and time again, as is made clear by the quote from earlier. If this really is all there is to the phenomenology of conscious pain, then it should also be all there is to the phenomenology of conscious thought. Either the theory works for thoughts or it doesn't work for sensations.

If one bites the bullet and stipulates that thoughts do not have phenomenology, then, as we have seen, the issue becomes merely verbal, about how we want to use certain words, or one is revealed as an eliminativist about consciousness. By insisting that one's theory is not committed to cognitive phenomenology one deepens the mystery as to how higher-order thoughts result in the kind of conscious mental life we actually experience, perhaps even to the point of intractability. Thus 
we arrive at the fundamental dilemma that faces those higher-order thought theorists who refuse to posit cognitive phenomenology: The more serious one takes the phenomenology of pain (or vision, or audition, etc.), the more pressing this problem becomes, while the less serious one takes that phenomenology, the more trivial the higher-order thought theory of consciousness begins to look.

We have here been focusing on Rosenthal's version of the theory and have explicitly adopted his construal of the term 'what it is like'. Though we haven't argued for it here, we think that these results can be straightforwardly extended to any interpretation of that phrase. So, if one accepts a higher-order thought theory and one wants to reject the identification what-it-is-likeness with mental appearances, the arguments of this paper would still suggest that (a) All phenomenology is fundamentally cognitive and (b) There is a distinctive, proprietary, perhaps individuative, phenomenology associated with conscious thoughts.

We conclude that whatever one's ultimate position is about the existence of cognitive phenomenology, in either the general or central sense, it looks like higherorder thought theories are committed to its existence. Whether one finds that a welcome result or a disastrous consequence is a matter for another discussion.

\section{ACKNOWLEDGMENTS}

We would like to thank David Rosenthal, Jake Berger, and David Pitt for highly detailed and helpful discussion of the issues in the paper. Thanks also are due to discussions of this material with Josh Weisberg, Steven Horst, Robert Barnard, and Michael Lynch, and to audiences of presentations at the Southern Society for Philosophy and Psychology and the American Philosophical Association.

\section{NOTES}

1. We are aware that there are those who question the usefulness of this terminology (Lycan 1996, 77, cf. 176 n. 3; Hellie 2007), yet it is common parlance among many working in this area (see Smithies 2011). In addition, since we aim to pose a challenge from within the HOT-theoretic framework we aim to use the phrase in the way that higher-order theorists, like David Rosenthal, actually use the phrase. As we spell this out in the next section, this is the sense in which it picks out mental appearances.

2. In addition we use the more cumbersome 'what it is like for one' phraseology both to keep in line with Rosenthal's usage and also to indicate that there is some kind of higher-order awareness involved in ordinary conscious experience (cf. Rosenthal 2002).

3. This is tendentious because it assumes that one can make a distinction between state consciousness and phenomenal consciousness such that it makes sense to say that the first-order state is state conscious (but not phenomenally conscious) while the higher-order thought itself is phenomenally conscious (but not state conscious). This is not the place to defend this claim and it is not clear that both authors endorse it, but see Brown 2012 and Lau and Brown forthcoming for a defense. 
4. For instance here is David Chalmers making a similar remark in an aside in his paper on the unity of consciousness. He says, "Rosenthal holds that only sensory states could be phenomenally conscious ... this is arguably merely a verbal difference, however, since Rosenthal holds that there will be something that it is like to be in a state whenever it is the object of the right sort of higherorder thought, whether that state is sensory or not" (Chalmers 2010, 531).

5. This kind of eliminativism is distinct from the kind explored by Mandik (in press). There Mandik is concerned with the issue of whether the first-order states need to exist or not. Here we are concerned with the question of whether conscious pains really feel like anything at all.

\section{REFERENCES}

Bayne, T., and M. Montague, eds. 2011. Cognitive Phenomenology. Oxford: Oxford University Press.

Block, N. 2011. “The Higher-Order Approach to Consciousness Is Defunct.” Analysis 71 (3): 419-31.

Brown, R. 2007. "The Mark of the Mental." Southwest Philosophy Review 23 (1): 117-24.

Brown, R. 2012. "The Brain and Its States." In Being in Time: Dynamical Models of Phenomenal Experience. Advances in Consciousness Research. Edited by Shimon Edelema, Tomer Fekete, and Neta Zach. Amsterdam, The Netherlands, and Philadelphia, PA: John Benjamins Press.

Hellie, B. 2007. “There's Something It's Like' and the Structure of Consciousness." Philosophical Review 116(3): 441-63. doi:10.1215/00318108-2007-005.

Lau, H., and R. Brown. Forthcoming. "The Emperor's New Phenomenology? The Empirical Case for Conscious Experience without First-Order Representations." In Themes from Block. Edited by Adam Pautz and Daniel Stoljar. Cambridge, MA: MIT Press.

Lycan, W. 1996. Consciousness and Experience. Cambridge, MA: MIT Press.

Mandik, Pete. In press. "Conscious-State Anti-realism." In Content and Consciousness Revisited. Edited by C. Munoz-Suarez and F. De Brigard. Berlin: Springer.

Nagel, T. 1974. "What Is It Like to Be a Bat?" Philosophical Review 83: 435-50.

Pitt, D. 2004. “The Phenomenology of Cognition or What Is It Like to Think That P?" Philosophy and Phenomenological Research 69(1).

Rosenthal, D. 1997. "A Theory of Consciousness." In The Nature of Consciousness: Philosophical Debates. Edited by Ned Block, Owen Flanagan, and Güven Güzeldere. Cambridge, MA: MIT Press/Bradford Books. 729-53.

Rosenthal, D. 2002. "How Many Kinds of Consciousness?” Consciousness and Cognition 11(4): $653-65$.

Rosenthal, D. 2005. Consciousness and Mind. Oxford: Oxford University Press.

Rosenthal, D. 2011. "Exaggerated Reports: Reply to Block." Analysis 71(3): 431-37.

Smithies, D. Forthcoming. "The Nature of Cognitive Phenomenology." Philosophy Compass. 